\title{
Brown bowel syndrome: an unusual cause of massive dilatation of the colon
}

\author{
M H E ROBINSON, B L DOWLING, J V CLARK, ANI) C H MASON \\ From the Departments of Surgery and Pathology, Northampton (General Hospital, Northampton
}

\begin{abstract}
summaкy We report a case of the brown bowel syndrome presenting as major dilatation of the colon which resembled 'toxic dilatation' and necessitated subtotal colectomy. We confirm the reported association between the brown bowel syndrome, malabsorption, and hypovitaminosis $\mathbf{E}$. Furthermore we document failure of the brown pigmentation to resolve after six months in spite of vitamin E supplements and correction of the malabsorption. Finally we suggest that, although the brown bowel syndrome is rare, it should be considered in cases of major colonic dilatation where the patient is or may be suffering from a malabsorption syndrome, and where the sigmoidoscopic appearances do not suggest severe inflammatory bowel disease.
\end{abstract}

Brown pigmentation of the muscular layers of the intestine (brown bowel syndrome, intestinal lipofuscinosis) has been described in association with deficiency of fat soluble vitamin $E$ occurring in various disorders causing malabsorption. In the absence of mechanical obstruction, major colonic dilatation occurring in a young patient usually occurs in association with severe inflammatory bowel disease. A combination of the brown bowel syndrome and colonic dilatation necessitated colectomy. This is previously undescribed.

\section{Case report}

A 34 year old man was admitted with a three week history of profuse watery diarrhoea, weight loss of three stone, anorexia and colicky lower abdominal pain. For two days he had had frank haematuria. Direct questioning revealed a history of episodic diarrhoca for years, mouth ulceration over the last year and sporadic nose bleeding for two months. He had no previous medical history of note and his only medication was diphenoxylate $5 \mathrm{mg}$ six hourly prescribed by his general practitioner two days before admission.

Physical examination revealed a cachectic man weighing $60 \mathrm{~kg}$. He was faintly icteric, afebrile, pulse 100 per minute, blood pressure 125/100. The

Address for correspondence: Mr M H F. Robinson. FRC S. Dept of Accident Scrvices. John Radeliffe Infirmary. Headington. Oxford.

Accepted for publication 7 Nowember lass. abdomen was distended and tympanic but nontender and bowel sounds were normal. The rectum

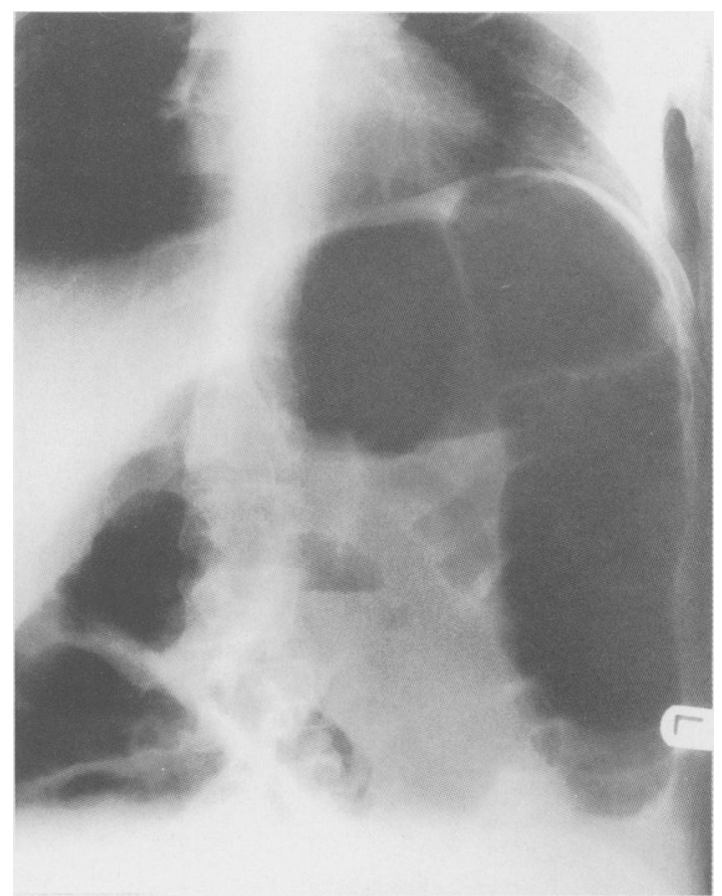

Fig. 1 Plain abdominal radiograph illustrating dilatation of the descending and transvere colon. 


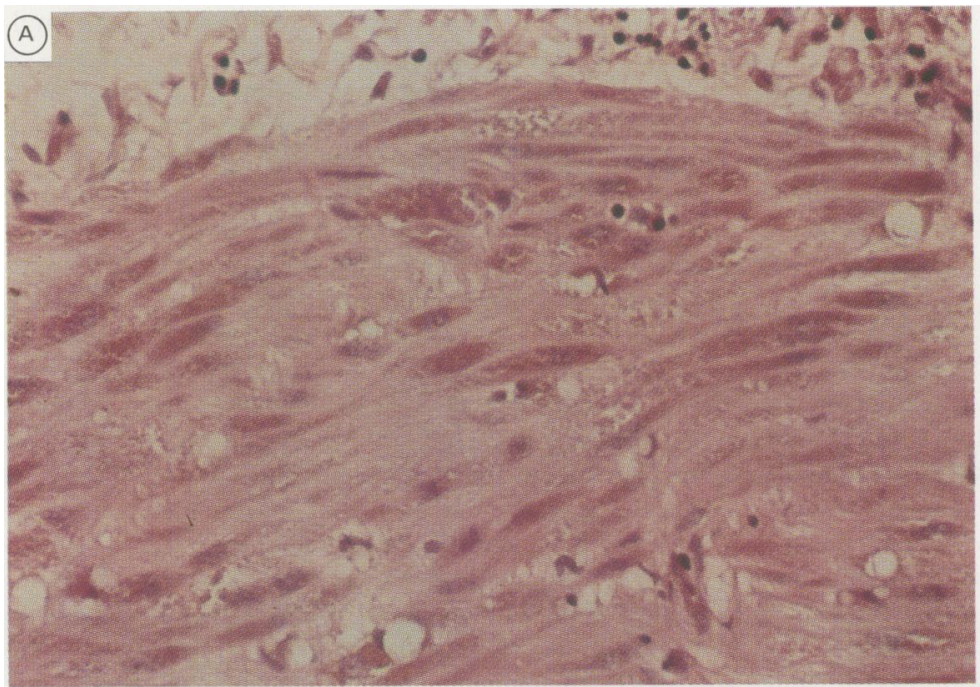

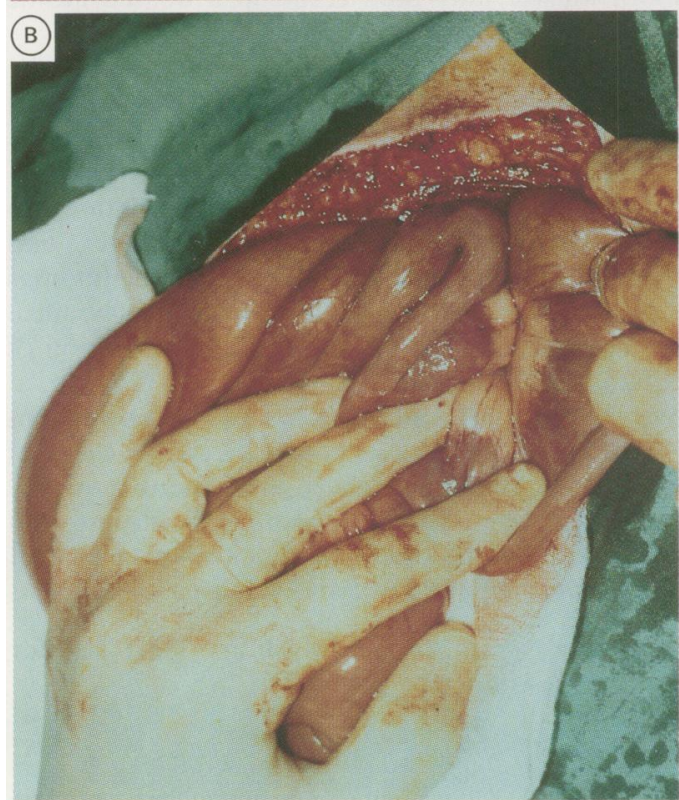

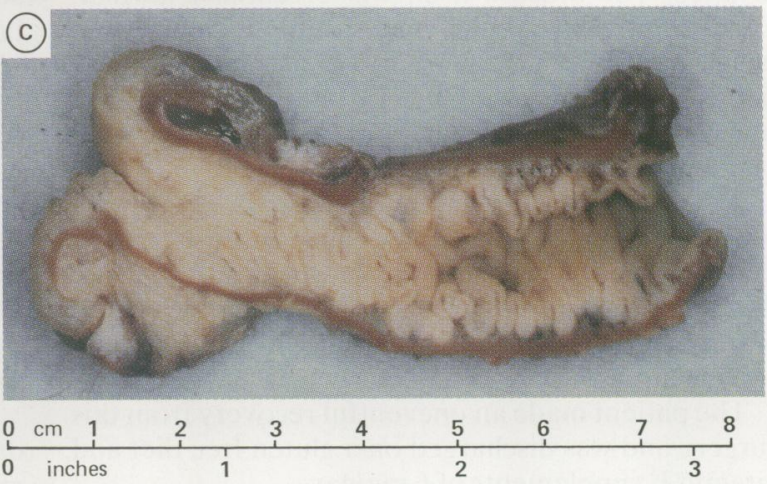

Fig. 2 (a) Brown lipofuscin granules within smooth muscle cells of the colonic muscularis propria. (b) Operative photograph illustrating the brown pigmentation of the bowel and its mesentery. (c) Resected ileostomy stump sectioned longitudinally to show brown pigmentation of the muscularis propria. was empty and sigmoidoscopy showed a granular but not grossly abnormal mucosa. Rectal biopsy showed a mild non-specific proctitis. The remainder of the examination was normal.

Blood tests were as follows: $\mathrm{Hb} 15.4 \mathrm{~g} / \mathrm{dl}$; WBC $13.7 \times 10^{9} / 1$; platelets $360 \times 10^{9} / \mathrm{l}$; ESR $26 \mathrm{~mm} / \mathrm{h}$. Urea and electrolytes normal; AST 67 IU/l (5-40); bilirubin $48 \mu \mathrm{mol} / \mathrm{l}(0-17)$; alkaline phosphatase 237 IU/l (30-135); CRP $58 \mathrm{mg} / \mathrm{l}(0-12)$. Prothrombin time $>180$ seconds (control 14 seconds); APTT $>240$ seconds (control 40 seconds); serum fibrinogen degradation products normal. Serum vitamin E $3 \cdot 0$ $\mu \mathrm{mol} / \mathrm{l}(>9 \cdot 0)$; serum vitamin A $3.3 \mu \mathrm{mol} / \mathrm{l}(0 \cdot 5-$ 4.4). Stool cultures showed no growth.

Plain abdominal radiographs showed $12 \mathrm{~cm}$ dilatation of the transverse and descending colon with thickening of the bowel wall (Fig. 1).

Treatment with intravenous steroids, antibiotics and total parenteral nutrition was started. He was given fresh frozen plasma and vitamin $\mathrm{K}$ in view of the deranged clotting and liver function tests. Limited barium enema revealed neither obstruction to the flow of contrast nor obvious mucosal abnormality. Serial plain radiographs showed no resolution 
of the megacolon and he underwent laparotomy four days after admission.

Laparotomy revealed a hugely dilated colon although the wall was neither fragile nor inflamed. There was no perforation. The colon and small bowel were stained brown throughout (Fig. 2b) though the small bowel was otherwise normal in appearance. Subtotal colectomy was performed and right iliac fossa ileostomy fashioned.

Microscopically (Fig. 2a), the smooth muscle cells of the muscularis propria of colon, appendix and terminal ileum were laden with granules of golden brown pigment. The pigment showed the histochemical staining properties of lipofuscin and also stained with a long Ziehl-Neelsen technique indicating the presence of ceroid. Pigment was present within clusters of macrophages within the muscularis propria and to a lesser extent within the muscularis mucosae and the tunica media of submucosal arteries. The mucosa itself was essentially normal. Extra cellular ovoid aggregates of identical pigment were noted within the sinusoids of mesenteric lymph nodes. Nerve fibres of the myenteric plexus were unusually prominent but contained no abnormal pigment and ganglion cells were normal.

A diagnosis of intestinal lipofuscinosis (brown bowel syndrome) was made and because of the known association with malabsorption a distal duodenal biopsy was performed. This showed the features of subtotal villous atrophy in keeping with coeliac disease.

The patient made an uneventful recovery from this surgery and was discharged on a gluten free diet and vitamin E supplements of $6 \mathrm{mg} /$ day.

Within six months the patient had regained his lost weight $(20 \mathrm{~kg})$ and the serum vitamin $E$ level had risen to just below the normal range $(7 \cdot 0 \mu \mathrm{mol} / \mathrm{l})$. In spite of this good clinical response to a gluten free diet, repeat distal duodenal biopsy showed no significant improvement in the villous pattern. Restorative ileorectal anastomosis was performed and at operation the macroscopic appearance of the bowel was unchanged. Microscopy of the excised ileostomy showed persisting intestinal lipofuscinosis. The pigment was visible macroscopically (Fig. 2c).

Postoperatively he made an uneventful recovery and has remained well since.

\section{Discussion}

Brown pigmentation of the bowel was first described by Wagner ${ }^{1}$ in 1861 , but its association with malabsorption and vitamin E deficiency was recognised initially by Pappenheimer and Victor' in 1946.

The origin of the pigment has been much debated but a convincing theory was proposed by Foster ${ }^{3}$ in 1979. He suggested that the brown bowel syndrome represents a smooth muscle mitochondrial myopathy analogous to the skeletal muscle mitochondrial myopathies and showed that the lipofuscin accumulates within effete smooth muscle mitochondria. Vitamin E exerts a high degree of conformational protection and environmental stability on the mitochondrial membranes and hence upon mitochondrial function by preventing membrane damage by free radicals released during oxidative phosphorylation. Lack of Vitamin E caused by malabsorption leads to peroxidation of unsaturated membrane lipids with accumulation of lipofuscin and results in mitochondrial malfunction. The loss of energy supply within the smooth muscle may then result in a dilated atonic bowel.

Our case illustrates major colonic dilatation in the brown bowel syndrome. This dilatation was sufficient to mimic the toxic dilatation seen in ulcerative colitis and Crohn's disease, and necessitated subtotal colectomy. This case confirms the relationship between malabsorption, hypovitaminosis $\mathrm{E}$, and brown bowel syndrome. In contrast with the findings of Lee et al, ${ }^{4}$ we show that a clinical and biochemical improvement may not be mirrored by resolution of the pathological lesion.

We suggest that, although the brown bowel syndrome is rare, it should be considered in cases of major colonic dilatation where the patient is or may be suffering from a malabsorption syndrome and where the sigmoidoscopic appearances do not suggest severe inflammatory bowel disease.

We are very grateful to Mrs Hilary Hart for her secretarial assistance.

\section{References}

1 Wagner E. Uber eine eigenthumliche primare Feltmetamorphose der Muskelhaut des Dunndarms. Arch der Heilkunde 1861; $2:$ 4.5.-9.

2 Pappenheimer AM, Victor a. Ceroid pigment in human tissues. Am J Pathol 1946; 22: 395-412.

3 Foster CS. The brown bowel syndrome: a possible smooth muscle mitochondrial myopathy. Histopathology 1979; 3: 1-17.

4 Lee SP. Nicholson GI. Ceroid enteropathy and Vitamin E deficiency. NZ Med J 1976; 83: 318-20. 